\title{
A Shannon-Runge-Kutta-Gill Method for Convection-Diffusion Equations
}

\author{
Xiaoming Duan, ${ }^{1}$ Jinsong Leng, ${ }^{1}$ Carlo Cattani, ${ }^{2}$ and Caiyun $\mathrm{Li}^{1}$ \\ ${ }^{1}$ School of Mathematical Sciences, University of Electronic Science and Technology of China, Chengdu, Sichuan 611731, China \\ ${ }^{2}$ Department of Mathematics, University of Salerno, Via Ponte Don Melillo, 84084 Fisciano, Italy \\ Correspondence should be addressed to Jinsong Leng; jslengjs@gmail.com
}

Received 20 January 2013; Accepted 5 February 2013

Academic Editor: Shengyong Chen

Copyright (c) 2013 Xiaoming Duan et al. This is an open access article distributed under the Creative Commons Attribution License, which permits unrestricted use, distribution, and reproduction in any medium, provided the original work is properly cited.

\begin{abstract}
A Shannon-Rugge-Kutta-Gill method for solving convection-diffusion equations is discussed. This approach transforms convection-diffusion equations into one-dimensional equations at collocations points, which we solve by Runge-Kutta-Gill method. A concrete example solved is used to examine the method's feasibility.
\end{abstract}

\section{Introduction}

Most of the physics phenomenon are stated in terms of partial differential equations (PDEs). Convection-diffusion equation is a kind of PDE which can be used in many science and technology fields, especially in image and signal procession such as image segmentation and the quickly stability of image processing. The numerical solution of convection-diffusion equations as an important subject has always attracted the attentions of the researchers for a long time.

The standard Galerkin finite-element method can solve the solution of the equations, but it is numerically unstable for small values of the diffusion parameter. So on the basis of this method, in [1] King and Krueger investigated the effect of a stabilized finite-element approximation and drew a conclusion that the stabilized system can provide accurate controllers. A novel multilevel particle methods and two complementary approaches are researched in [2]. In this paper, a new class of particle based on mapping functions is introduced, and particle remeshing is used as a key element in overlapping domains in the particle-AMR method. For the two-dimensional convection-diffusion equation, Gupta et al. proposed a fourth-order nine-point compact finite-difference formulae, which is shown to be computationally efficient and stable and yield highly accurate numerical solutions in $[3,4]$. The resulting linear system is solved by classical iterative methods for large values of the Reynolds number in [4]. With the wavelet method, Shi et al. solved the solution of covectiondiffusion equations by Haar wavelet method in [5]. In short, convection-diffusion equations are studied by scholars via different methods.

Recently, Wavelet analysis as a new subject has attracted a lot of attention. As a mathematical tool, it has been widely used in numerical analysis, signal processing $[6,7]$, image processing, and so forth. Many years ago, wavelet methods were used for numerical analysis, particularly the numerical solution of PDEs. Up to now, researchers have utilized the simplest Haar wavelets to solve kinds of PDEs. Chen and Hsiao, in [8], established an operational matrix of integration based on Haar wavelets and used a procedure for applying the matrix to obtain wavelet solution of PDEs. In $[9,10]$, Cattani solved Poisson's problem and Fredholm type integral equations by Harmonic wavelet method. Other wavelets are also extensively used to solve the kinds of PDEs, in which Shannon wavelet is applied in the numerical solution of some equations, such as $[11,12]$. Shannon scaling function and Sinc function combined with other methods (Galerkin, etc.) are used to solve some PDEs $[13,14]$. In light of the above description, we are enlightened that Shannon wavelet is a useful tool to obtain the solution of convection-diffusion equations, which combined with Rugge-Kutta-Gill method.

In this paper, the content is assigned as follows. In Section 2, Shannon wavelet is introduced. We elaborate the concrete method solving convection-diffusion equation in 
Section 3. In Section 4 the viability of Shannon wavelet collocation is tested by a listed example.

\section{Preliminaries}

2.1. Shannon Wavelet. Wavelets are classified as families with names, such as Haar wavelet, Meyer wavelet, and Shannon wavelet. Shannon wavelets are the real part of harmonic wavelets. They have a slow decay in the time domain but a very sharp compact support in the frequency (Fourier) domain. This fact, together with the Parseval's identity, is used to compute the inner product and the expansion coefficients of the Shannon wavelets easily. A set of Shannon scaling functions in the subspace $V_{j}$ is defined as

$$
\varphi_{j, k}(t)=2^{j / 2} \frac{\sin \pi\left(2^{j} t-k\right)}{\pi\left(2^{j} t-k\right)}, \quad k \in \mathbf{Z}
$$

and the mother wavelets are

$$
\begin{aligned}
& \psi_{j, k}(t) \\
& =2^{j / 2} \frac{\sin \pi\left(2^{j} t-k-1 / 2\right)-\sin 2 \pi\left(2^{j} t-k-1 / 2\right)}{\pi\left(2^{j} t-k-1 / 2\right)},
\end{aligned}
$$

In (1) and (2), the scaling function and mother wavelet for $j=k=0$ (see Figure 1) are as follows:

$$
\begin{gathered}
\varphi(t)=\frac{\sin \pi t}{\pi t}=\frac{e^{\pi i t}-e^{-\pi i t}}{2 \pi i t}, \\
\psi(t)=\frac{\sin \pi(t-1 / 2)-\sin 2 \pi(t-1 / 2)}{\pi(t-1 / 2)} .
\end{gathered}
$$

To some properties of Shannon scaling and wavelet functions, Cattani has detailedly researched in [15-18]. So in this paper, we will not narrate the properties again.

To (3), its Fourier transform $\hat{\varphi}(\omega)=\chi_{[-1 / 2,1 / 2)}$. It is very easy to see that

$$
\sum_{n=-\infty}^{\infty}|\widehat{\varphi}(\omega+n)|^{2}=1
$$

According to this equation, the sequence of function $\{\varphi(x-n)\}_{n=-\infty}^{\infty}$ is orthonormal. A reproducing kernel is generated [19] as follows:

$$
K(x-y)=\frac{\sin \pi(x-y)}{\pi(x-y)} .
$$

Recomposing (6), we obtain a new reproducing kernel

$$
w(x-y)=\frac{\sin (\pi / \Delta)(x-y)}{(\pi / \Delta)(x-y)},
$$

where $\Delta$ is the spatial mesh size.
In one-dimensional function $f(x)$, we make the domain $[a, b]$ be discrete and set the grid size

$$
\Delta=\frac{b-a}{2^{j}}
$$

So we obtain collocation points

$$
x_{i}=i \Delta, \quad i=0,1,2, \ldots, 2^{j}
$$

where $2^{j}$ is a number of nodes, which used in the discretization and also is the maximum wavelet index number. Now, a basis function $w_{j}\left(x-x_{n}\right)$ of Shannon wavelet will be constructed by (7)

$$
w_{j}\left(x-x_{n}\right)=\frac{\sin (\pi / \Delta)\left(x-x_{n}\right)}{(\pi / \Delta)\left(x-x_{n}\right)}, \quad n=0,1,2, \ldots, 2^{j}
$$

It has some properties as follows.

(i) To the random $x_{k}\left(k=0,1,2, \ldots, 2^{j}\right)$, the function $w_{j}\left(x-x_{n}\right)$ fulfills interpolation property:

$$
w_{j}\left(x_{k}-x_{n}\right)=\delta_{k n}= \begin{cases}1 & k=n \\ 0 & k \neq n\end{cases}
$$

(ii) We have noticed that the constructed basis functions are orthogonal to each other as follows:

$$
\int_{-\infty}^{\infty} w_{j}\left(x-x_{k}\right) w_{j}\left(x-x_{n}\right) d x=\Delta \delta_{k n}
$$

(iii) If we make the integral with the basis functions and their derived functions, we obtain.

$$
\int_{-\infty}^{\infty} w_{j}\left(x-x_{k}\right) \frac{d^{m} w_{j}\left(x-x_{n}\right)}{d x^{m}} d x=\Delta \frac{d^{m} w_{j}\left(x_{k}-x_{n}\right)}{d x^{m}}
$$

Both $w_{j}\left(x-x_{k}\right)$ and its associated wavelet play an important part in signal processing. Unfortunately, when $x \rightarrow \infty$, the reduction of $w_{j}\left(x-x_{k}\right)$ is very slow. So our paper only researches the case which $x$ belongs to finite interval.

2.2. Function Approximation. According to Shannon's sampling theorem, any function $f(x) \in B_{\pi}^{2}$ can be denoted as [19]

$$
f(x)=\sum_{n \in Z} f\left(x_{n}\right) w_{j}\left(x-x_{n}\right)
$$

where the coefficients $f\left(x_{n}\right)$ is the value of the function $f(x)$ at the point $x_{n} . B_{\pi}^{2}$ is the Paley-Wiener reproducing kernel Hilbert space which is a subspace of the Hilbert space $L^{2}(R)$. 

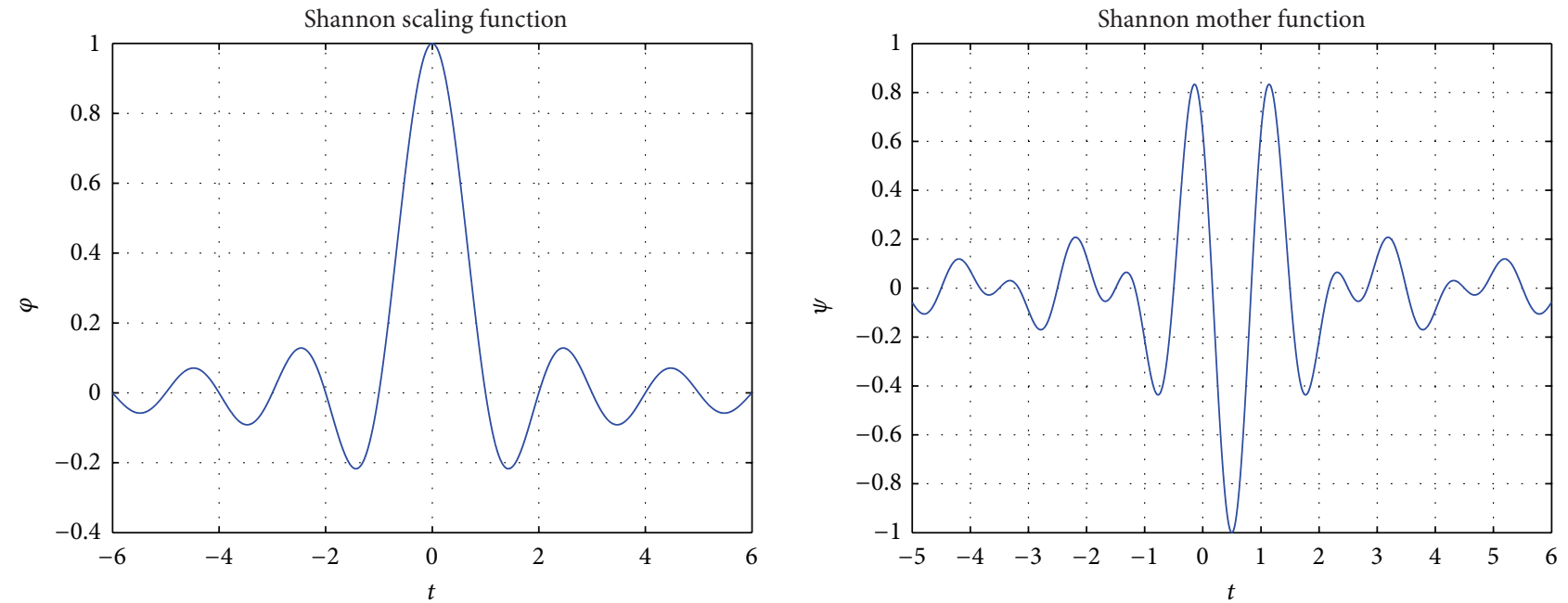

FIGURE 1: Shannon scaling function $\varphi$ and mother function $\psi$.
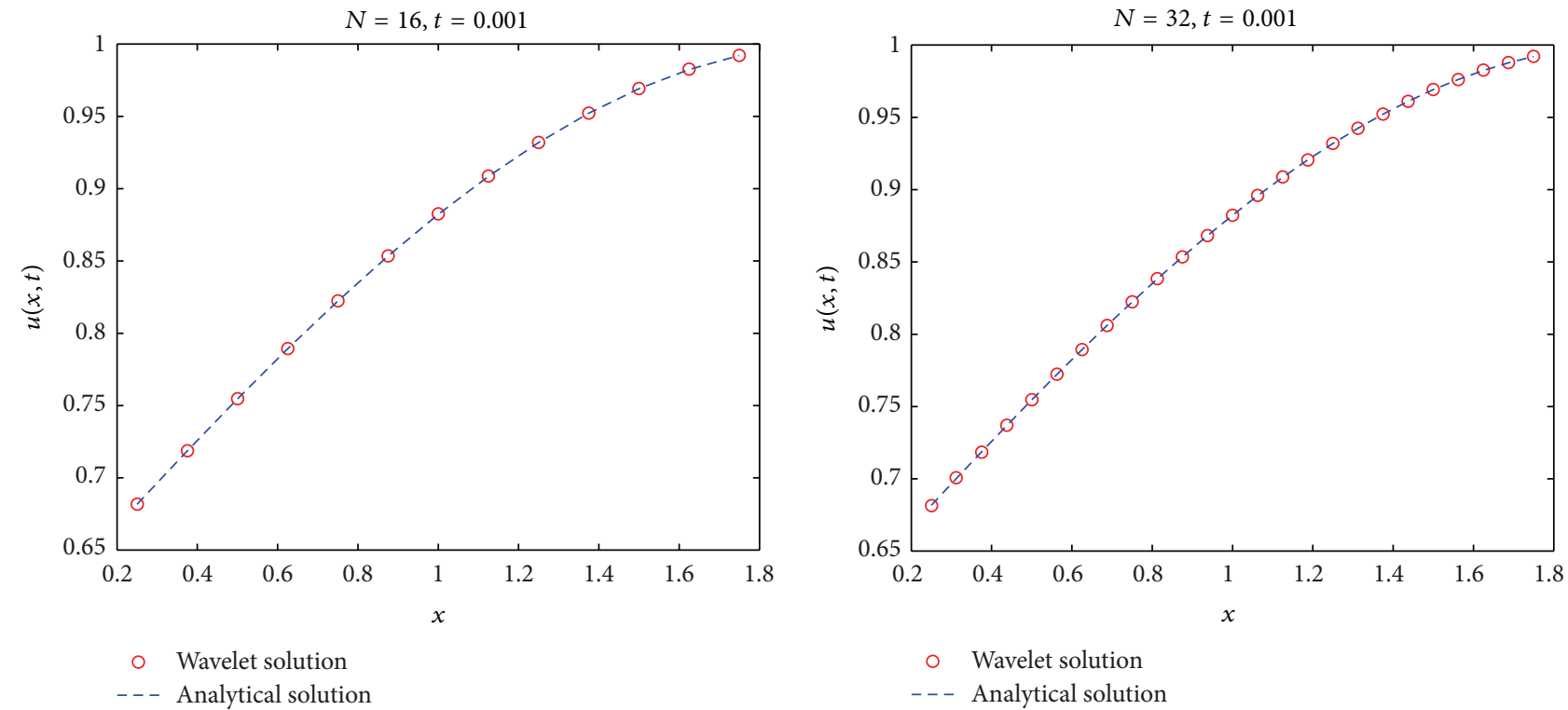

FIgURE 2: Comparison of the analytical solution and Shannon wavelet solution.

In $V_{j}, f(x)$ can be approximated by $f_{j}(x) \in V_{j}$. So we get

$$
f(x) \approx f_{j}(x)=\sum_{n=0}^{2^{j}} f_{j}\left(x_{n}\right) w_{j}\left(x-x_{n}\right) .
$$

\section{Method of Solution of Convection-Diffusion Equation}

In this section, let us consider the one-dimensional convection-diffusion equation with constant coefficients:

$$
\frac{\partial u}{\partial t}+a \frac{\partial u}{\partial x}=\alpha \frac{\partial^{2} u}{\partial x^{2}} \quad 0<x<2,0<t<T
$$

with initial condition and boundary conditions:

$$
\begin{gathered}
u(x, 0)=f(x), \quad 0 \leq x \leq b, \\
u(0, t)=g_{0}(t), \quad u(2, t)=g_{1}(t), \quad 0<t \leq T .
\end{gathered}
$$

Like (8) and (9), we will also divide the interval $[0,2]$ into $N=2^{j}$ equal parts of length $\Delta=2 / N$ and denote $x_{i}=i \Delta, i=$ $0,1,2, \ldots, N$. We know that $u(x, t)$ can be approximated by $u_{j}(x, t) \in V_{j}$ expanded in terms of the constructed basis function as formula (15) as follows:

$$
u(x, t) \approx u_{j}(x, t)=\sum_{n=0}^{N} u_{j}\left(x_{n}, t\right) w_{j}\left(x-x_{n}\right) .
$$


We multiply formula (16) with the constructed basis function $w_{j}\left(x-x_{k}\right)$, then we obtain

$$
\begin{aligned}
& \frac{\partial u_{j}(x, t)}{\partial t} w_{j}\left(x-x_{k}\right) \\
& \quad=-a \frac{\partial u_{j}(x, t)}{\partial x} w_{j}\left(x-x_{k}\right)+\alpha \frac{\partial^{2} u_{j}(x, t)}{\partial x^{2}} w_{j}\left(x-x_{k}\right)
\end{aligned}
$$

Integrate that formula (19) with respect to $x$ from $-\infty$ to $\infty$ as follows:

$$
\begin{aligned}
\int_{-\infty}^{\infty} & \frac{\partial u_{j}(x, t)}{\partial t} w_{j}\left(x-x_{k}\right) d x \\
= & \int_{-\infty}^{\infty}-a \frac{\partial u_{j}(x, t)}{\partial x} w_{j}\left(x-x_{k}\right) d x \\
& +\int_{-\infty}^{\infty} \alpha \frac{\partial^{2} u_{j}(x, t)}{\partial x^{2}} w_{j}\left(x-x_{k}\right) d x .
\end{aligned}
$$

The left expression of formula (20) is as follows:

$$
\begin{aligned}
\int_{-\infty}^{\infty} & \frac{\partial u_{j}(x, t)}{\partial t} w_{j}\left(x-x_{k}\right) d x \\
= & \int_{-\infty}^{\infty} \frac{\partial \sum_{n=0}^{N} u_{j}\left(x_{n}, t\right) w_{j}\left(x-x_{n}\right)}{\partial t} w_{j}\left(x-x_{k}\right) d x \\
= & \sum_{n=0}^{N} \frac{\partial u_{j}\left(x_{n}, t\right)}{\partial t} \int_{-\infty}^{\infty} w_{j}\left(x-x_{n}\right) w_{j}\left(x-x_{k}\right) d x \\
= & \frac{\partial \sum_{n=0}^{N} u_{j}\left(x_{n}, t\right) w_{j}\left(x_{k}-x_{n}\right)}{\partial t} \Delta \\
= & \frac{\partial u_{j}\left(x_{k}, t\right)}{\partial t} \Delta .
\end{aligned}
$$

The right expression of formula (20) is as follows:

$$
\begin{aligned}
\int_{-\infty}^{\infty} & -a \frac{\partial \sum_{n=0}^{N} u_{j}\left(x_{n}, t\right) w_{j}\left(x-x_{n}\right)}{\partial x} w_{j}\left(x-x_{k}\right) d x \\
& +\int_{-\infty}^{\infty} \alpha \frac{\partial^{2} \sum_{n=0}^{N} u_{j}\left(x_{n}, t\right) w_{j}\left(x-x_{n}\right)}{\partial x^{2}} w_{j}\left(x-x_{k}\right) d x \\
= & -a \sum_{n=0} N u_{j}\left(x_{n}, t\right) \int_{-\infty}^{\infty} \frac{\partial w_{j}\left(x-x_{n}\right)}{\partial x} w_{j}\left(x-x_{k}\right) d x \\
& +\alpha \sum_{n=0} N u_{j}\left(x_{n}, t\right) \int_{-\infty}^{\infty} \frac{\partial^{2} w_{j}\left(x-x_{n}\right)}{\partial x^{2}} w_{j}\left(x-x_{k}\right) d x \\
= & -a \Delta \sum_{n=0} N u_{j}\left(x_{n}, t\right) w_{j}^{\prime}\left(x_{k}-x_{n}\right) \\
+ & \alpha \Delta \sum_{n=0} N u_{j}\left(x_{n}, t\right) w_{j}^{\prime \prime}\left(x_{k}-x_{n}\right) .
\end{aligned}
$$

Via $(21)=(22)$, we obtain the following expression:

$$
\frac{\partial u_{j}\left(x_{k}, t\right)}{\partial t}=\sum_{n=0}^{N} u_{j}\left(x_{n}, t\right)\left[-a w_{j}^{\prime}\left(x_{k}-x_{n}\right)+\alpha w_{j}^{\prime \prime}\left(x_{k}-x_{n}\right)\right]
$$

To any $x_{k}$, we will get one equation. So $N+1$ equations will be obtained. In order to simplify the $N+1$ equations, we define the matrices $U$ and $V$ as follows:

$$
\begin{gathered}
U=\left[u_{j}\left(x_{0}, t\right), u_{j}\left(x_{1}, t\right), u_{j}\left(x_{2}, t\right), \ldots, u_{j}\left(x_{N}, t\right)\right]^{\top}, \\
v_{k n}=-a w_{j}^{\prime}\left(x_{k}-x_{n}\right)+\alpha w_{j}^{\prime \prime}\left(x_{k}-x_{n}\right), \\
V=\left(v_{k n}\right)_{(N+1) \times(N+1)} .
\end{gathered}
$$

Combining with (24), the formula (23) is evolved into a matrix equation:

$$
\frac{\partial U}{\partial t}=V U
$$

Now we will use Runge-Kutta-Gill method to solve formula (25) as follows:

$$
\begin{gathered}
U_{i+1}=U_{i}+\frac{\Delta t}{6}\left[K_{1}+(2-\sqrt{2}) K_{2}+(2+\sqrt{2}) K_{3}+K_{4}\right], \\
K_{1}=V U_{i} \\
K_{2}=V\left(U_{i}+\frac{1}{2} K_{1}\right) \\
K_{3}=V\left(U_{i}+\frac{\sqrt{2}-1}{2} K_{1}+\frac{2-\sqrt{2}}{2} K_{2}\right) \\
K_{4}=V\left(U_{i}-\frac{\sqrt{2}}{2} K_{2}+\frac{2+\sqrt{2}}{2} K_{3}\right)
\end{gathered}
$$

where $\Delta t$ is the time interval. From (9) and (17), the initial value $U_{0}$ is obtained, and then we can evaluate the numerical solution at any collocation point within the different parameter $t$.

\section{Test of Example}

A concrete convection-diffusion equation has known exact solution will be considered, and we observe how well the Shannon wavelet solution approximates the exact solution. We assume that $\alpha=0.1, a=0.8$,

$$
\begin{gathered}
f(x)=e^{-(x-2)^{2} / 8}, \\
g_{0}(x)=\sqrt{\frac{20}{20+t}} e^{-(5+4 t)^{2} / 10(t+20)}, \\
g_{1}(t)=\sqrt{\frac{20}{20+t}} e^{-2(5+2 t)^{2} / 5(t+20)},
\end{gathered}
$$


For which the exact solution is

$$
u(x, t)=\sqrt{\frac{20}{20+t}} e^{-(x-2-0.8 t)^{2} / 0.4(t+20)} .
$$

In the course of the experiment, we got $t_{\max }=0.01$ and setted $\Delta t=0.00001$. We got the approximate charts in the case of $N=32$ and $N=16$ and obtained the conclusion that the wavelet solution is approximate to the exact solution more precisely (see Figure 2).

\section{Conclusion}

In this paper, the theory of Shannon wavelet combined with Runge-Kutta-Gill method is used to solve the approximation of convection-diffusion equations. It has been shown that the key idea of shannon wavelet collocation method is to transform convection-diffusion equations into one-dimensional equations at collocations points and to solve the problem via Rugge-Kutta-Gill method.

\section{Acknowledgment}

The authors are thankful to the referees for their valuable comments and suggestions that improved the presentation of this paper. This work was supported by the National Natural Science Foundation of China (11271001).

\section{References}

[1] B. B. King and D. A. Krueger, "The 1-D convection diffusion equation: galerkin least squares approximations and feedback control," in Proceedings of the 43rd IEEE Conference on Decision and Control, pp. 1502-1507, 2004.

[2] M. Bergdorf, G.-H. Cottet, and P. Koumoutsakos, "Multilevel adaptive particle methods for convection-diffusion equations," Multiscale Modeling and Simulation, vol. 4, no. 1, pp. 328-357, 2005.

[3] M. M. Gupta, R. P. Manohar, and J. W. Stephenson, "A fourth order, cost effective and stable finite difference scheme for the convection-diffusion equation," in Numerical Properties and Methodologies in Heat Transfer, pp. 201-209, Hemisphere, 1983.

[4] M. M. Gupta, R. P. Manohar, and J. W. Stephenson, "A single cell high order scheme for the convection-diffusion equation with variable coefficients," International Journal for Numerical Methods in Fluids, vol. 4, no. 7, pp. 641-651, 1984.

[5] Z. Shi, L. Y. Deng, and Q. J. Chen, "Numerical solution of differential equations by using Haar wavelets," in Proceedings of the International Conference on Wavelet Analysis and Pattern Recognition, pp. 2-4, Beijing, China, 2007.

[6] C. Cattani, "Wavelet based approach to fractals and fractal signal denoising," Transactions on Computational Sciences Journal, vol. 5730, pp. 143-162, 2009.

[7] J. S. Leng, T. Z. Hung, Y. F. Jing, and W. Jiang, "A study on conjugate quadrature filters," EURASIP Journal on Advances in Singnal Processing, vol. 2011, Article ID 231754, 7 pages, 2011.

[8] C. F. Chen and C. H. Hsiao, "Haar wavelet method for solving lumped and distributedparameter systems," IEE ProceedingsControl Theory and Applications, vol. 144, pp. 87-94, 1997.
[9] C. Cattani, "Harmonic wavelet solution of Poisson's problem," Balkan Journal of Geometry and Its Applications, vol. 13, no. 1, pp. 27-37, 2008.

[10] C. Cattani and A. Kudreyko, "Harmonic wavelet method towards solution of the Fredholm type integral equations of the second kind," Applied Mathematics and Computation, vol. 215, no. 12, pp. 4164-4171, 2010.

[11] C. Cattani, "Shannon wavelets for the solution of integrodifferential equations," Mathematical Problems in Engineering, vol. 2010, Article ID 408418, 22 pages, 2010.

[12] C. Cattani, "Second order Shannon wavelet approximation of $C^{2}$-functions," Politehnica University of Bucharest Scientific Bulletin A, vol. 73, no. 3, pp. 73-84, 2011.

[13] V. G. Koures, "Solving the Coulomb Schrödinger equation in $d=2+1$ via sinc collocation," Journal of Computational Physics, vol. 128, no. 1, pp. 1-5, 1996.

[14] F. Stenger, "A "sinc-Galerkin" method of solution of boundary value problems," Mathematics of Computation, vol. 33, no. 145, pp. 85-109, 1979.

[15] C. Cattani, "Connection coefficients of Shannon wavelets," Mathematical Modelling and Analysis, vol. 11, no. 2, pp. 117-132, 2006.

[16] C. Cattani, "Shannon wavelets theory," Mathematical Problems in Engineering, vol. 2008, Article ID 164808, 24 pages, 2008.

[17] C. Cattani, "Signorini cylindrical waves and Shannon wavelets," Advances in Numerical Analysis, vol. 2012, Article ID 731591, 24 pages, 2012.

[18] C. Cattani, "Fractional calculus and Shannon wavelets," Mathematical Problems in Engineering, vol. 2012, Article ID 502812, 26 pages, 2012.

[19] G. W. Wei, "Quasi wavelets and quasi interpolating wavelets," Chemical Physics Letters, vol. 296, pp. 215-222, 1998. 


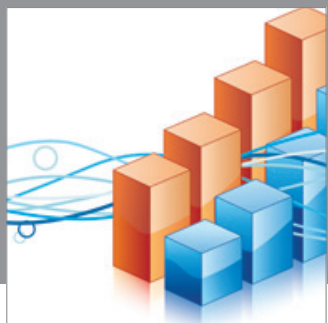

Advances in

Operations Research

mansans

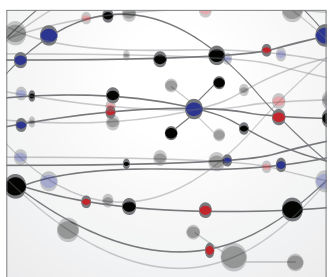

The Scientific World Journal
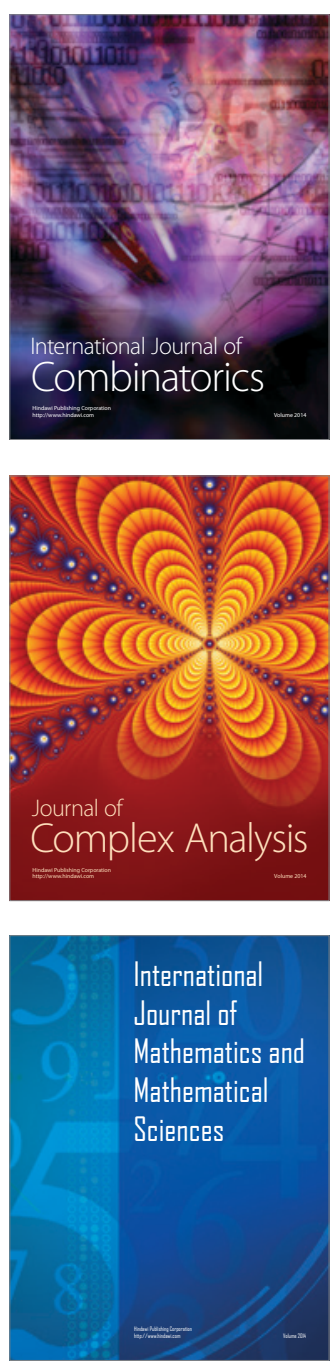
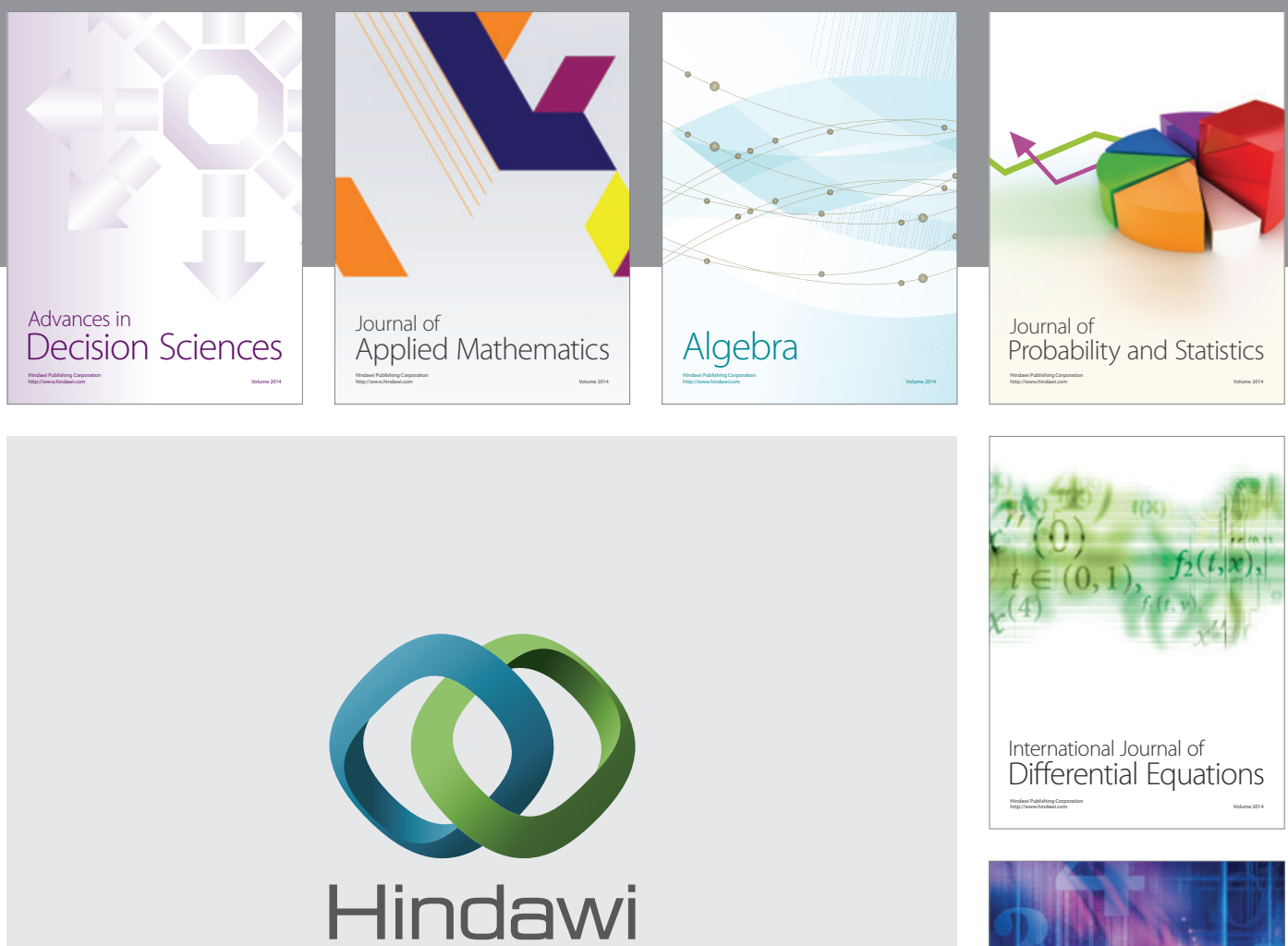

Submit your manuscripts at http://www.hindawi.com
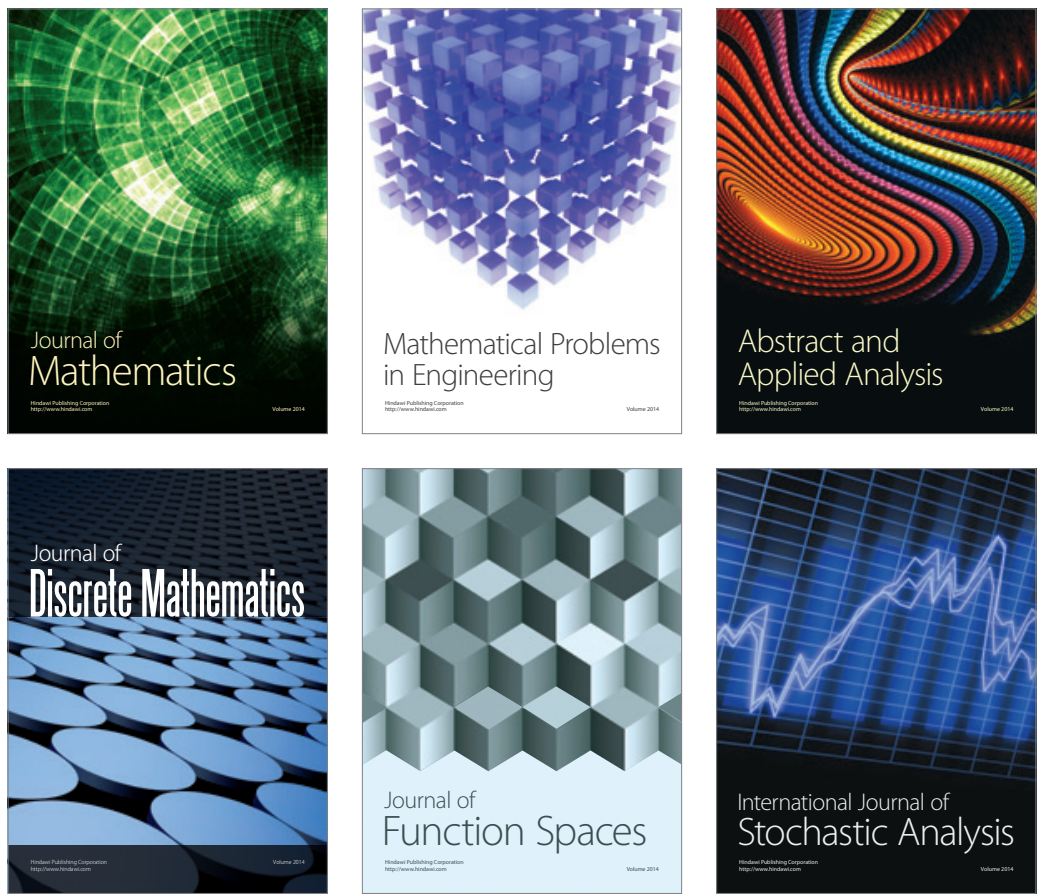

Journal of

Function Spaces

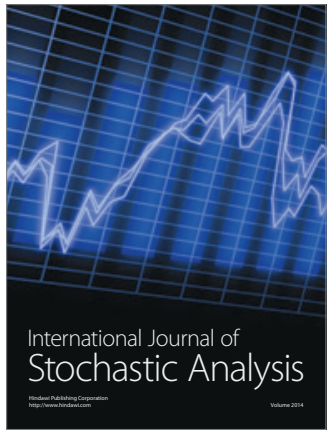

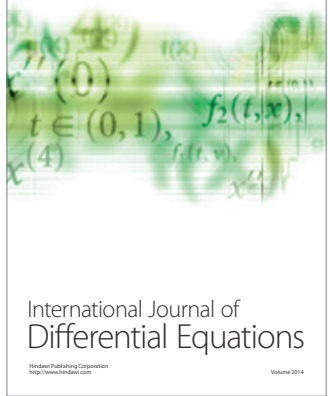
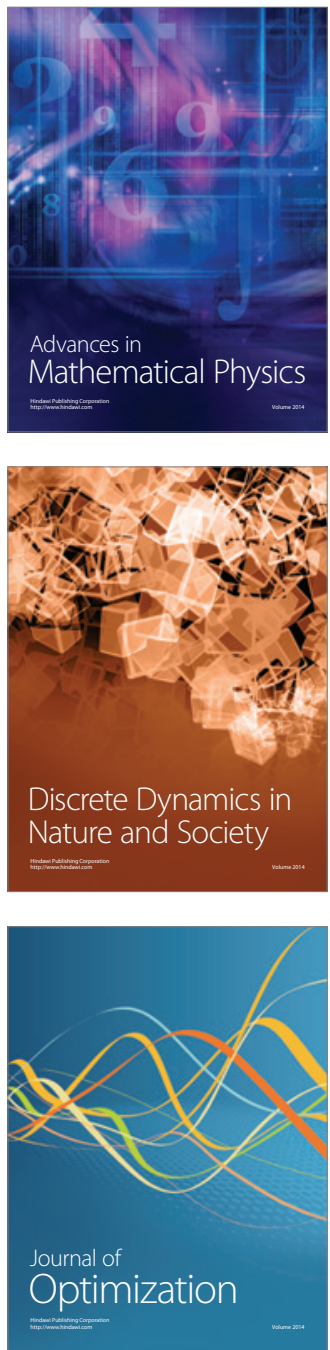